PROCEEDINGS OF THE

AMERICAN MATHEMATICAL SOCIETY

Volume 138, Number 6, June 2010, Pages 2181-2185

S 0002-9939(10)10239-1

Article electronically published on January 28, 2010

\title{
THE UNIQUENESS OF SYMMETRIZING MEASURE OF MARKOV PROCESSES
}

\author{
JIANGANG YING AND MINZHI ZHAO
}

(Communicated by Richard C. Bradley)

\begin{abstract}
In this short article, we shall introduce the notion of fine irreducibility and give some of its equivalent statements. Then we prove that the fine irreducibility implies the uniqueness of symmetrizing measures for a right Markov process.
\end{abstract}

\section{INTRODUCTION}

Let us now introduce some background on Markov processes. Let $X=\left(X_{t}, \mathbf{P}^{x}\right)$ be a right Markov process on a state space, which is usually assumed to be Radon, with transition semigroup $\left(P_{t}\right)$. It is a bit difficult to make clear what a right Markov process means, but roughly it is a right continuous process with the strong Markov property. The interested readers may refer to Sharpe's book 4 for details. A $\sigma$-finite measure on $E$ is called a symmetrizing measure for $X$ or $\left(P_{t}\right)$ if

$$
\int_{E} P_{t} f(x) g(x) m(d x)=\int_{E} f(x) P_{t} g(x) m(d x)
$$

for each non-negative measurable $f, g$ and $t>0$. Of course, neither existence nor uniqueness of symmetrizing measures is guaranteed, and it is certainly one of the most interesting and fundamental problems for a Markov process. For a discrete time Markov chain with countable state space, Kolmogorov's cycle test actually gives a necessary and sufficient condition for existence and uniqueness. However there has been no counterpart for general Markov processes. This article aims to give a sufficient condition for the uniqueness of the symmetrizing measure.

\section{The UNIQUENESS OF SYMMETRIZING MEASURE}

We first present a theorem which states a condition for the uniqueness of the symmetrizing measure and will be used later. This kind of results may be known in some other forms. We begin with a general right Markov process $X=\left(X_{t}, \mathbf{P}^{x}\right)$

Received by the editors July 24, 2009, and, in revised form, September 30, 2009.

2010 Mathematics Subject Classification. Primary 60J45, 60J40.

Key words and phrases. Symmetrizing measure, linear diffusion, Dirichlet space, regular subspace.

The research of the first author was supported in part by NSFC Grant No. 10771131.

The research of the second author was supported in part by NSFC Grant No. 10601047.

(C)2010 American Mathematical Society
public domain 28 years from publication 
on the state space $E$ (with its Borel $\sigma$-field $\mathscr{B}(E)$ ) with transition semigroup $\left(P_{t}\right)$ and resolvent operator $\left(U^{\alpha}\right)$,

$$
\begin{aligned}
U^{\alpha} 1_{A}(x) & =U^{\alpha}(x, A)=\mathbf{E}^{x} \int_{0}^{\infty} e^{-\alpha t} 1_{A}\left(X_{t}\right) d t \\
& =\int_{0}^{\infty} e^{-\alpha t} P_{t}(x, A) d t .
\end{aligned}
$$

For any subset $A$ of $E, T_{A}$ denotes the first hitting time of $A$, which is a stopping time if $A$ is nearly Borel. A subset $D$ of $E$ is finely open if

$$
\mathbf{P}^{x}\left(T_{D^{c}}>0\right)=1
$$

for any $x \in D$; i.e., any point in $D$ will stay in $D$ for a positive period of time. The topology containing all finely open sets is called the fine topology related to $X$. Since $X$ is right continuous, any open set must be finely open and hence the fine topology is finer than the usual topology.

It is easy to see from the right continuity that for $x \in E$ and a finely open subset $D, \mathbf{P}^{x}\left(T_{D}<\infty\right)>0$ if and only if $U^{\alpha} 1_{D}(x)>0$. The process $X$ is called finely irreducible if $\mathbf{P}^{x}\left(T_{D}<\infty\right)>0$ for any $x \in E$ and a non-empty finely open subset $D$, where $T_{D}$ is the hitting time of $D$. Intuitively the fine irreducibility means that any point can reach any non-empty finely open set, while the usual irreducibility means that any point can reach any non-empty open set. Certainly the fine irreducibility is stronger than the usual irreducibility. Since the fine irreducibility is hard to characterize, we shall give a few equivalent statements which may be useful in some circumstances.

Lemma 2.1. The following statements are equivalent.

(1) $X$ is finely irreducible.

(2) $U^{\alpha} 1_{D}$ is positive everywhere on $E$ for any non-empty finely open set $D$.

(3) $U^{\alpha} 1_{A}$ is either identically zero or positive everywhere on $E$ for any Borel set $A$ or, in other words, $\left\{U^{\alpha}(x, \cdot): x \in E\right\}$ are all mutually absolutely continuous.

(4) All non-trivial excessive measures are mutually absolutely continuous.

Proof. The equivalence of (1) and (2) is easy. We shall prove that they are equivalent to (3). We may assume $\alpha=0$. Suppose (1) is true. If $U 1_{A}$ is not identically zero, then there exists $\delta>0$ such that $D:=\left\{U 1_{A}>\delta\right\}$ is non-empty. Since $U 1_{A}$ is excessive and thus finely continuous, $D$ is finely open and the fine closure of $D$ is contained in $\left\{U 1_{A} \geq \delta\right\}$. Then

$$
U 1_{A}(x) \geq P_{D} U 1_{A}(x)=\mathbf{E}^{x}\left(U 1_{A}\left(X_{T_{D}}\right)\right) \geq \delta \mathbf{P}^{x}\left(T_{D}<\infty\right)>0 .
$$

Conversely suppose (3) is true. Then for any finely open set $D$, by the right continuity of $X, U 1_{D}(x)>0$ for any $x \in D$. Therefore $U 1_{D}$ is positive everywhere on $E$.

Let $\xi$ be an excessive measure. Since $\alpha \xi U^{\alpha} \leq \xi, \xi(A)=0$ implies that $\xi U^{\alpha}(A)=$ 0 . However $\xi$ is non-trivial. Thus it follows from $(3)$ that $U^{\alpha} 1_{A} \equiv 0$, i.e., that $A$ is potential zero. Conversely if $A$ is potential zero, then $\xi(A)=0$ for any excessive measure $\xi$. Therefore (3) implies (4).

Assume $(4)$ holds. Since $U(x, \cdot)$ is excessive for all $x$, they are equivalent. This implies (3). 
A Borel set $A$ is said to be of potential zero if $U^{\alpha} 1_{A}$ is identically zero for some $\alpha \geq 0$ (thus for all $\alpha \geq 0$ ). A $\sigma$-finite measure $\mu$ on $E$ is said to be a symmetrizing measure of $X$ or $X$ is said to be $\mu$-symmetric if

$$
\left(P_{t} u, v\right)_{\mu}=\left(u, P_{t} v\right)_{\mu}
$$

for any measurable $u, v \geq 0$ and $t>0$. It is easy to check that any symmetrizing measure is excessive and an excessive measure does not charge any set of potential zero.

Theorem 2.1. Assume that $X$ is finely irreducible. Then the symmetrizing measure of $X$ is unique up to a constant. More precisely, if both $\mu$ and $\nu$ are non-trivial symmetrizing measures of $X$, then $\nu=c \mu$ with a positive constant $c$.

Proof. First of all there exists a measurable set $H$ such that both $\mu(H)$ and $\nu(H)$ are positive and finite, because $\mu$ and $\nu$ are equivalent by Lemma 2.1. This is actually true when both measures are $\sigma$-finite and one is absolutely continuous with respect to another. Indeed, assume that $\nu \ll \mu$. Since $\nu$ is non-trivial and $\sigma$-finite, we may find a measurable set $B$ such that $0<\nu(B)<\infty$. Then $\mu(B)>0$. Since $\mu$ is $\sigma$-finite, there exist $A_{n} \uparrow E$ such that $0<\mu\left(A_{n}\right)<\infty$. Then $\nu\left(A_{n} \cap B\right) \uparrow \nu(B)$ and $\mu\left(A_{n} \cap B\right) \uparrow \mu(B)$. Hence there exists some $n$ such that $\nu\left(A_{n} \cap B\right)>0$. Take $H=A_{n} \cap B$, which makes both $\mu(H)$ and $\nu(H)$ positive and finite.

Set $c=\nu(H) / \mu(H)$. We may assume that $c=1$ without loss of generality. Let $m=\mu+\nu$. Then there are $f_{1}, f_{2} \geq 0$ such that $\mu=f_{1} \cdot m$ and $\nu=f_{2} \cdot m$. Let $A=\left\{f_{1}>f_{2}\right\}, B=\left\{f_{1}=f_{2}\right\}$ and $C=\left\{f_{1}<f_{2}\right\}$.

We shall show that $\nu=\mu$. Otherwise $\mu(A)>0$ or $\nu(C)>0$. We assume that $\mu(A)>0$ without loss of generality. Since $\mu$ is $\sigma$-finite, there is $A_{n} \in \mathscr{B}(E)$ such that $A_{n} \subseteq A, \mu\left(A_{n}\right)<\infty$ and $A_{n} \uparrow A$. Let $D=B \cup C$. For any integer $n$ and $\alpha>0$,

$$
\left(U^{\alpha} 1_{A_{n}}, 1_{D}\right)_{\mu} \leq\left(U^{\alpha} 1_{A_{n}}, 1_{D}\right)_{\nu}=\left(U^{\alpha} 1_{D}, 1_{A_{n}}\right)_{\nu} \leq\left(U^{\alpha} 1_{D}, 1_{A_{n}}\right)_{\mu} .
$$

Since $\left(U^{\alpha} 1_{A_{n}}, 1_{D}\right)_{\mu}=\left(U^{\alpha} 1_{D}, 1_{A_{n}}\right)_{\mu}$, it follows that $\left(U^{\alpha} 1_{D}, 1_{A_{n}}\right)_{\nu}=\left(U^{\alpha} 1_{D}, 1_{A_{n}}\right)_{\mu}$. Thus we have

$$
\left(U^{\alpha} 1_{D},\left(1-\frac{f_{2}}{f_{1}}\right) 1_{A_{n}}\right)_{\mu}=\left(U^{\alpha} 1_{D}, 1_{A_{n}}\right)_{\mu}-\left(U^{\alpha} 1_{D}, 1_{A_{n}}\right)_{\nu}=0 .
$$

Since $1-\frac{f_{2}}{f_{1}}>0$ on $A$, let $n$ go to infinity; and by the monotone convergence theorem we get that $\left(U^{\alpha} 1_{D}, 1_{A}\right)_{\mu}=0$. The fine irreducibility of $X$ implies that $U^{\alpha} 1_{D}=0$ identically or $D$ is of potential zero. Therefore

$$
\mu(D)=\nu(D)=0 .
$$

Consequently,

$$
0=\mu(H)-\nu(H)=\int_{H \cap A}\left(1-\frac{f_{2}}{f_{1}}\right) d \mu,
$$

which leads to that $\mu(H \cap A)=0$ and also $\mu(H)=0$. The contradiction implies that $\nu=\mu$.

The following example shows that the usual irreducibility is not enough to guarantee the uniqueness of the symmetrizing measure, while the fine irreducibility might be too strong. 
Example. Let $J=\frac{1}{4}\left(\delta_{1}+\delta_{-1}+\delta_{\sqrt{2}}+\delta_{-\sqrt{2}}\right)$ be defined on $\mathbb{R}$ and $\pi=\left\{\pi_{t}\right\}_{t>0}$ be the corresponding convolution semigroup; i.e., $\hat{\pi}_{t}(x)=e^{-t \phi(x)}$ with

$$
\phi(x)=\int(1-\cos x y) J(d y)=\frac{1}{2}(1-\cos x)+\frac{1}{2}(1-\cos \sqrt{2} x) .
$$

Let $X$ be the corresponding Lévy process. Then $X$ is symmetric with respect to the Lebesgue measure. Let $N=\{n+m \sqrt{2}: n, m$ are integers $\}$ and $\mu=\sum_{x \in N} \delta_{x}$. Then $\mu$ is $\sigma$-finite and also a symmetrizing measure. It is easy to see that any point $x$ can reach any point in $x+N$ and cannot reach outside of $x+N$. Since $x+N$ is dense in $\mathbb{R}$, any point can reach any non-empty open set; namely, $X$ is irreducible. However any compound Poisson process will stay at the starting point for a positive period of time; i.e., any singleton is finely open. Hence $X$ is not finely irreducible.

Another interesting example is also a compound Poisson process $X$, where the Lévy measure $J$ is a probability measure on $\mathbb{R}$ with a continuous even density. In this case, we can show that $X$ has a unique symmetrizing measure, the Lebesgue measure, but $X$ is still irreducible, while not finely irreducible. Actually any point cannot reach any other single point.

It is known that the fine topology is determined by the process and hard to identify usually. Hence it is hard to verify sometimes the fine irreducibility defined in the theorem. However under LSC, namely, assuming that $U^{\alpha} 1_{B}$ is lower-semicontinuous for any Borel subset $B$ of $E$, the fine irreducibility is equivalent to the usual one, which is easier to verify: $\mathbf{P}^{x}\left(T_{D}<\infty\right)>0$ for any $x \in E$ and non-empty open subset $D \subset E$. In fact, take $A \in \mathscr{B}(E)$ with $U^{\alpha} 1_{A} \neq 0$ identically. There is $b>0$ such that $G=\left\{U^{\alpha} 1_{A}>b\right\} \neq \emptyset$ and is open due to the property LSC. Again by Proposition II.2.8 in [1], for any $x \in E$,

$$
U 1_{A}(x) \geq P_{G} U 1_{A}(x)=\mathbf{P}^{x}\left(U 1_{A}\left(X_{T_{G}}\right), T_{G}<\infty\right) .
$$

But $X_{T_{G}} \in \bar{G}$ on $\left\{T_{G}<\infty\right\}$ by Theorem I.11.4 in [1] and then $X_{T_{G}} \geq b$ on $\left\{T_{G}<\infty\right\}$. Hence by the irreducibility, we have

$$
U 1_{A}(x) \geq b \mathbf{P}^{x}\left(T_{G}<\infty\right)>0 .
$$

It is obvious that the strong Feller property and the irreducibility imply the fine irreducibility.

Remark. As a remark, we would like to present a slightly more general result which was provided by Masatoshi Fukushima in his comment to this theorem.

Suppose that $X$ is $\mu$-symmetric. The following two definitions refer to Definition 2.1.1 in [2]. A Borel subset $A$ is called $\left(P_{t}\right)$-invariant if $1_{A} \cdot P_{t}\left(1_{A^{c}} f\right)=0$ a.e. $\mu$ for all $t>0$ and $f \in L^{2}(E, \mu)$, and $X$ is $\mu$-irreducible if any $\left(P_{t}\right)$-invariant set is trivial in the sense that either $\mu(A)=0$ or $\mu\left(A^{c}\right)=0$. Then the following statements are equivalent due to Theorem 3.5.6 in 2 and a similar proof of Lemma 2.1 .

(1) $X$ is $\mu$-irreducible;

(2) if $D$ is finely open and $\mu(D)>0$, then $\mathbf{P}^{x}\left(T_{D}<\infty\right)>0$ for q.e. $x \in E$;

(3) $U^{\alpha} 1_{D}>0$ q.e. for any finely open $D$ with $\mu(D)>0$;

(4) $U^{\alpha} 1_{A}$ is either 0 q.e. or positive q.e. for every Borel subset $A$.

It follows that if $X$ is $\mu$-irreducible, then all non-trivial excessive measures charging no $\mu$-polar sets are equivalent. Hence following the proof of Theorem 2.1 we have the version stated below. 
Theorem 2.2. Assume that a Borel right process $X$ is $\mu$-irreducible with respect to some non-trivial symmetrizing measure $\mu$ of $X$. If $\nu$ is a symmetrizing measure of $X$ charging no $\mu$-polar sets, then $\nu=c \cdot \mu$ for some constant $c \geq 0$.

\section{ACKNOWLEDGEMENT}

The authors would like to thank Professor Masatoshi Fukushima for his useful comments.

\section{REFERENCES}

[1] R. Blumental and R. K. Getoor, Markov Processes and Potential Theory, Academic Press, New York, 1968. MR0264757 (41:9348)

[2] Z. Q. Chen and M. Fukushima, Symmetric Markov Processes, Time Change and Boundary Theory, available at http://www.math.washington.edu/ zchen/CF/cfbook-PUP32.pdf.

[3] M. Fukushima, Y. Oshima and M. Takeda, Dirichlet Forms and Symmetric Markov Processes, Walter de Gruyter, Berlin, 1994. MR1303354 (96f:60126)

[4] M. J. Sharpe, General Theory of Markov Processes, Academic Press, Boston, MA, 1988. MR958914(89m:60169)

Department of Mathematics, Fudan University, Shanghai 200433, People's Republic OF CHINA

Department of Mathematics, Zhejiang University, Hangzhou 310027, People's RepubLIC OF CHINA 